\title{
On-Surface Synthesis of Unsaturated Carbon Nanostructures with Regularly Fused Pentagon-Heptagon Pairs
}

\author{
Ian Cheng-Yi Hou," Qiang Sun," Kristjan Eimre, Marco Di Giovannantonio, José I. Urgel, \\ Pascal Ruffieux, Akimitsu Narita,* Roman Fasel,* and Klaus Müllen*
}

Cite This: J. Am. Chem. Soc. 2020, 142, 10291-10296

Read Online

\section{ACCESS | Llll Metrics \& More | 回 Article Recommendations | (s) Supporting Information}

ABSTRACT: Multiple fused pentagon-heptagon pairs are frequently found as defects at the grain boundaries of the hexagonal graphene lattice and are suggested to have a fundamental influence on graphene-related materials. However, the construction of $\mathrm{sp}^{2}$ carbon skeletons with multiple regularly fused pentagon-heptagon pairs is challenging. In this work, we found that the pentagonheptagon skeleton of azulene was rearranged during the thermal reaction of an azulene-incorporated organometallic polymer on $\mathrm{Au}(111)$. The resulting $\mathrm{sp}^{2}$-carbon frameworks were characterized by high-resolution scanning probe microscopy techniques and feature novel polycyclic architectures composed of multiple regularly fused pentagon-heptagon pairs. Moreover, the calculated analysis of its aromaticity revealed a peculiar polar electronic structure.

$\mathrm{T}$ the hexagonal lattice of graphene, nanostructures of fused 1 pentagon-heptagon pairs are found as defects at grain boundaries (see Figure 1a,b). ${ }^{1-6}$ Numerous calculations have

a

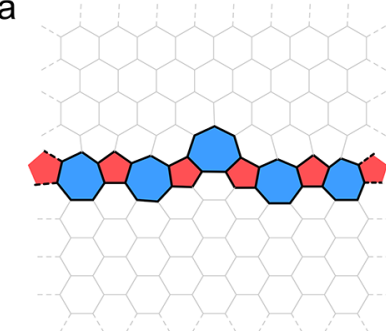

C

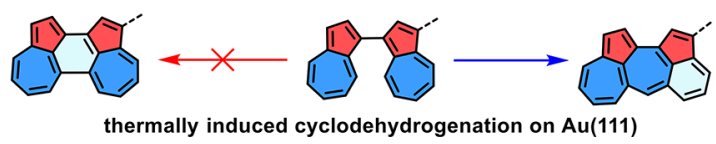

Figure 1. Schematic representation of fused pentagon-heptagon pairs at graphene (a) grain boundary ${ }^{4}$ and (b) grain boundary loop ${ }^{1}$ and (c) the formation of fused pentagon-heptagon pairs during cyclodehydrogenation of oligo(1,3-azulenediyl) discovered in this work.

suggested that controlling these "defect" structures is essential for tuning the properties of graphene-based materials. ${ }^{5,7-15}$ The unsaturated pentagon-heptagon bicyclic hydrocarbon, named azulene, is a classic of organic chemistry. ${ }^{16}$ Organic synthesis has also provided several polycyclic aromatic hydrocarbons (PAHs) containing two fused azulenes, which exhibit unique physical properties, including small optical energy gaps and open-shell biradical characters. ${ }^{17-24}$ Nevertheless, the construction of $\mathrm{sp}^{2}$-carbon skeletons with multiple fused pentagon-heptagon pairs has remained challenging.
On-surface synthesis is emerging as a new method with remarkable success in fabricating novel $\mathrm{sp}^{2}$-carbon nanostructures that are often inaccessible by conventional organic synthesis. ${ }^{25-30}$ Typically, molecular precursors are sublimed onto metal surfaces under ultrahigh vacuum (UHV) conditions, followed by thermally induced surface-catalyzed reactions. ${ }^{25,31,32}$ Syntheses of PAHs containing two embedded pentagon-heptagon pairs in their skeletons have been sporadically explored employing cyclodehydrogenation of precursors composed mainly of hexagons on $\mathrm{Au}(111)$ at elevated temperature. ${ }^{33-35}$ On-surface polymerizations ${ }^{36-39}$ and strain-induced ${ }^{40}$ or thermally activated ${ }^{38}$ rearrangements of azulene derivatives have also been reported. Polymers with multiple azulene units could serve as potential precursors for polycyclic $\mathrm{sp}^{2}$-carbon nanostructures with abundant pentagon-heptagon pairs. Recently, it has been shown that poly(2,6-azulenediyl) can undergo lateral fusion on $\mathrm{Au}(111)$ when annealed at high temperature. ${ }^{38}$ Nevertheless, among all these reported on-surface synthesized $\mathrm{sp}^{2}$-carbon nanostructures, heptagon-pentagon pairs are isolated from each other by hexagons.

During our investigation of utilizing 1, $1^{\prime}$-dibromo-3,3'biazulenyl (2) on $\mathrm{Au}(111)$ for the synthesis of poly $(1,3,4,8$ azulenetetrayl) (6), a nonlinear graphene nanoribbon analogue with a nonhexagonal skeleton (Figure 1c and Scheme 1), its thermally induced reactions remained without success. Instead, novel $\mathrm{sp}^{2}$-carbon nanostructures were formed featuring cyclopenta $[e f]$ indeno $[7,1-b c]$ heptalene subunits as chain ends

Received: April 2, 2020

Published: May 19, 2020

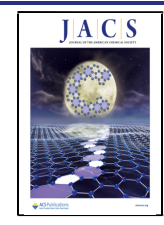


Scheme 1. Synthesis of 2 in Solution, Its Polymerization on $\mathrm{Au}(111)$, and Further Thermal Reaction ${ }^{a}$

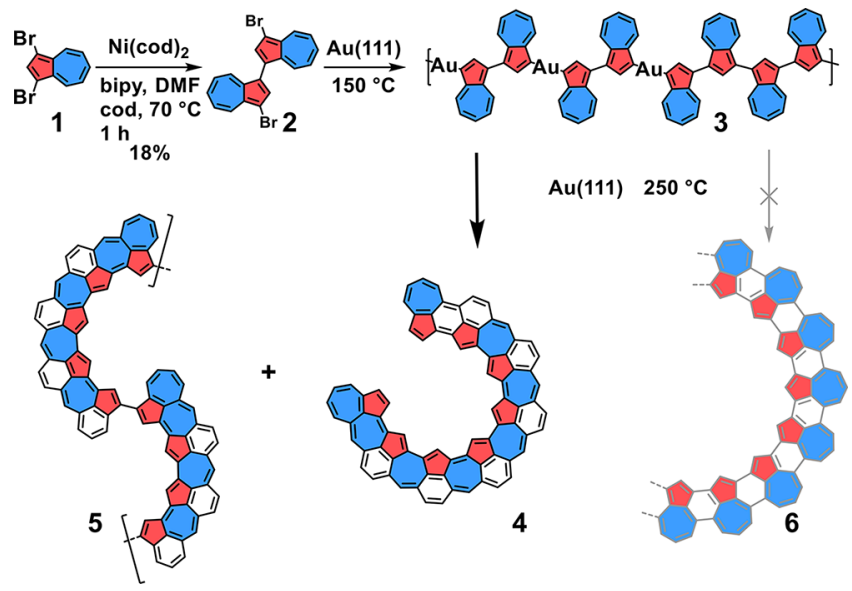

${ }^{a}$ Reagents: $\operatorname{cod}=1,5$-cyclooctadiene, bipy $=2,2^{\prime}$-bipyridine, DMF $=$ dimethylformamide.

(Figure 1c), together with a polycyclic skeleton composed of multiple regularly fused pentagon-heptagon pairs (Scheme 1). One such nanostructure with 23 polygons was unambiguously characterized by means of high-resolution scanning tunneling microscopy (STM) and noncontact atomic force microscopy (nc-AFM). Additionally, its aromaticity was analyzed by nucleus-independent chemical shifts (NICS) and anisotropy of the induced current density (ACID) calculations. The formation of this unique architecture presumably involved a radical-promoted domino skeletal rearrangement of azulenediyl moieties. This finding appears promising for the on-surface synthesis of new types of carbon-based materials.

First, molecular precursor $\mathbf{2}$ was synthesized under Yamamoto conditions that have been already used previously for the polymerization ${ }^{41}$ of 1,3 -dibromoazulene ${ }^{42}$ (1) in solution. The stoichiometry of the $\mathrm{Ni}(\mathrm{cod})_{2}$ reagent was controlled to suppress the formation of higher oligomers.
Biazulenyl 2 was isolated as the major product in $18 \%$ yield, accompanied by $25 \%$ recovery of $\mathbf{1}$ (Scheme 1 ). Then, compound 2 was deposited under UHV on $\mathrm{Au}(111)$ held at room temperature. The molecules self-assembled into aggregates, presumably stabilized by halogen-halogen interactions $^{43}$ (Figure S1). Upon further annealing at $150{ }^{\circ} \mathrm{C}$, debrominative polymerization of $\mathbf{2}$ was triggered, leading to the formation of polymer chains (Scheme 1), which were confirmed by STM (Figure 2a). In a close-up image (Figure 2b), 1,1'-biazulenyl-3,3'-diyl ${ }^{44}$ moieties were resolved, and we assigned the bright protrusions to heptagons (Figure 2c, indicated by green arrows). This interpretation was further supported by nc-AFM imaging, where the heptagons are more clearly resolved than the pentagons (Figure $2 \mathrm{~d}$ ). Considering the large distance $(\sim 1 \mathrm{~nm})$ and protrusions between most of the neighboring 1,1'-biazulenyl-3,3'-diyl moieties (indicated by red arrows in Figure $2 \mathrm{c}-\mathrm{e})$, the observed polymer 3 is attributed to a 1,1'-biazulenyl-3,3'-diyls-Au organometallic polymer. ${ }^{36,37}$ Although $\mathrm{C}-\mathrm{Au}-\mathrm{C}$ bonds are rarely formed on $\mathrm{Au}(111)$, they seem to occur where steric hindrance between debrominated species hampers subsequent $\mathrm{C}-\mathrm{C}$ bond formation. ${ }^{45}$ Interestingly, despite abundant $\mathrm{C}-\mathrm{Au}-\mathrm{C}$ motifs in 3 , some $\mathrm{C}-\mathrm{C}$ bonds were already formed, as evidenced by the existence of oligo(1,3-azulenediyl) moieties in the polymer chain (Figure 2c-e).

After further annealing of the sample at $250{ }^{\circ} \mathrm{C}$, STM images disclosed drastically different curved nanostructures (Figure $3 \mathrm{a}, \mathrm{b}, \mathrm{e})$. Nc-AFM imaging was employed to resolve a short segment (4) consisting of 23 polygons (Figure 3c). Its skeleton displayed multiple fused pentagon-heptagon pairs composed solely of carbon atoms with no residual $\mathrm{Au}$ atoms. Notably, despite the chain ends featuring a cyclopenta[ef]indeno[7,1$b c]$ heptalene and an isolated azulene skeleton, all pentagons (highlighted in red) appeared in the inner part of the curved structure, with the hexagons (in white) staying in the outer and the heptagons (in blue) in the middle parts (Figure 3c,d). Shorter segments with 17 polygons presenting the same structural feature were also observed (Figure S2). Note that we
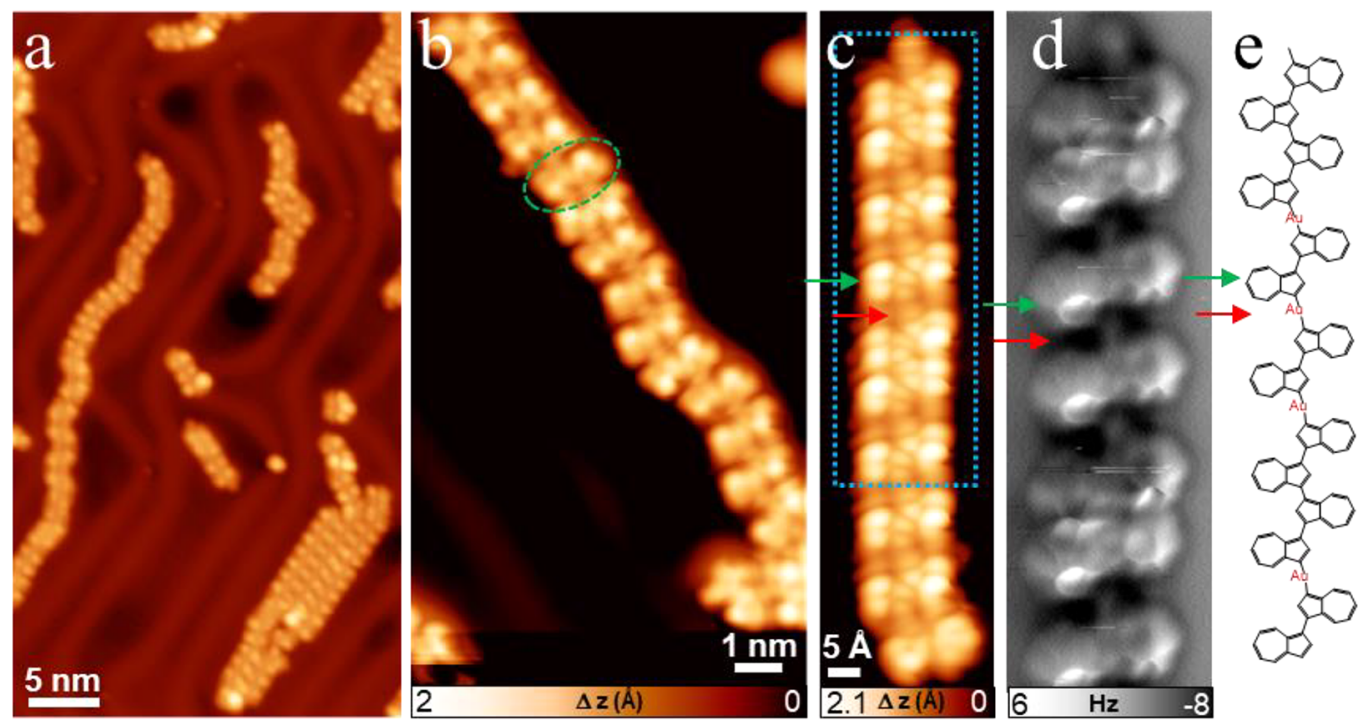

Figure 2. (a) An STM image after annealing 2 on $\mathrm{Au}(111)$ at $150{ }^{\circ} \mathrm{C}\left(V_{\mathrm{s}}=-1.5 \mathrm{~V}, \mathrm{I}_{\mathrm{t}}=0.07 \mathrm{nA}\right)$ and (b) a close-up STM image of organometallic polymer $3\left(V_{\mathrm{s}}=-0.1 \mathrm{~V}, I_{\mathrm{t}}=0.11 \mathrm{nA}\right)$. A biazulenyldiyl moiety is marked by a green ellipse. (c) A high-resolution STM image of an organometallic chain imaged by a CO-functionalized tip $\left(V_{\mathrm{s}}=-0.05 \mathrm{~V}, I_{\mathrm{t}}=0.1 \mathrm{nA}\right)$. (d) A bond-resolved nc-AFM image of the marked region in $(\mathrm{c})\left(V_{\mathrm{s}}=-5\right.$ $\mathrm{mV}$, oscillation amplitude: $\sim 80 \mathrm{pm}$ ) and its (e) assigned chemical structure. 

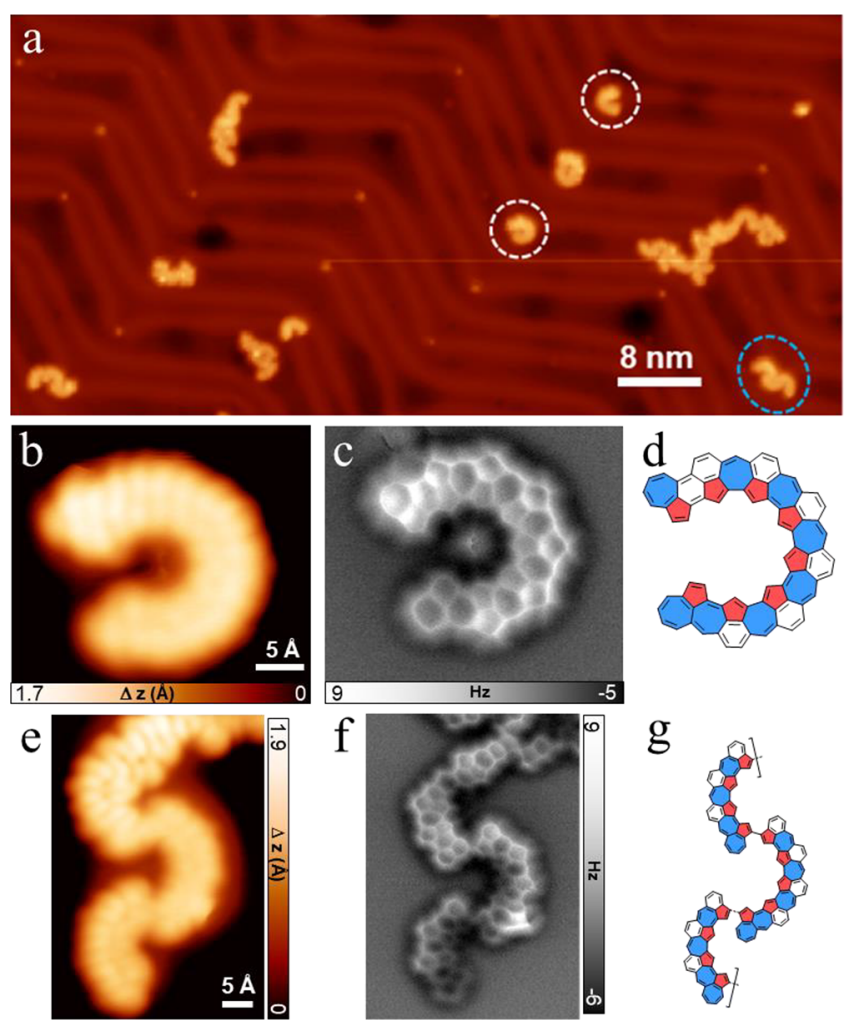

Figure 3. (a) An STM image after further annealing 3 at $250{ }^{\circ} \mathrm{C}\left(V_{s}=\right.$ $\left.-1 \mathrm{~V}, I_{\mathrm{t}}=0.1 \mathrm{nA}\right)$. (b) A close-up STM image of $4\left(V_{\mathrm{s}}=-0.02 \mathrm{~V}, I_{\mathrm{t}}=\right.$ $0.1 \mathrm{nA})$ and its (c) bond-resolved nc-AFM image $\left(V_{s}=-5 \mathrm{mV}\right.$, oscillation amplitude: $\sim 80 \mathrm{pm}$ ) and (d) chemical structure. (e) A close-up STM image of polymer $5\left(V_{s}=-0.02 \mathrm{~V}, I_{\mathrm{t}}=0.1 \mathrm{nA}\right)$ and its (f) bond-resolved nc-AFM image $\left(V_{\mathrm{s}}=-5 \mathrm{mV}\right.$, oscillation amplitude: $\sim 80 \mathrm{pm})$ and $(\mathrm{g})$ illustrative chemical structure. Nanostructures circled in (a) correspond to (b,c) (white) and (e,f) (blue).

did not find fully closed nanorings (Figure S3). However, there were several nanostructures (5) arising from polymeric precursors (Figure 3a). In the enlarged STM image, polygons linked by $\mathrm{C}-\mathrm{C}$ single bonds can be recognized (Figure $3 \mathrm{e}-\mathrm{g}$ ). Importantly, the conventional cyclodehydrogenation of oligo(1,3-azulenediyl)s would lead to oligo(1,3,4,8-azulenetetrayl)s
6 (Scheme S1). The formation of 4 and polymer 5 must therefore involve rearrangements of the azulene skeleton.

Recent studies have shown that azulene in a polymer chain hardly rearranges on $\mathrm{Au}(111)$ below $400{ }^{\circ} \mathrm{C} .{ }^{38-40}$ However, azulene slowly transforms into naphthalene when heated above $330{ }^{\circ} \mathrm{C} .{ }^{46}$ Similar reaction is also observed during the flashvacuum pyrolysis of azulene at $1000{ }^{\circ} \mathrm{C} .{ }^{47}$ The process is believed to proceed by intermediate radical adducts through "methylene walk" and "spiran" pathways. The azulene skeleton would thereby react from pentagon-heptagon to pentagonhexagon and eventually to hexagon-hexagon pairs. ${ }^{48-51}$ It is also reported that azulene interact more strongly with a metal surface than its isomer naphthalene. ${ }^{52,53}$ In our present work, we assume that oligo(1,3-azulenediyl)s were first formed by thermally induced $\mathrm{Au}$ extrusion from polymer 3 at elevated temperature (Scheme S1). Then, initial surface-assisted $\mathrm{C}-\mathrm{H}$ bond cleavage occurred primarily at azulenyl end-groups since they possess higher conformational mobility, which facilitates their interactions with the Au surface (Scheme S1). Next, the formed radical species attacked the neighboring azulenediyl moieties, triggering a chain reaction through repetitive steps of homoallyl-cyclopropylcarbinyl-radical-rearrangement/1,2-hydrogen-shift/interazulenediyl-radical-attack (Scheme S1). This sequence would eventually lead to an $\mathrm{sp}^{2}$-carbon skeleton with multiple fused pentagon-heptagon pairs. Density functional theory (DFT) calculations suggest that 4 is $3.05 \mathrm{eV}$ more stable than its counterpart octa(1,3,4,8-azulenetetrayl) on $\mathrm{Au}(111)$ substrate, revealing a driving force for the rearrangement to occur (Figure S4).

Compound 4 possesses an energy gap as low as $0.72 \mathrm{eV}$ calculated by DFT. Its highest occupied and lowest unoccupied molecular orbital exhibit larger lobes on opposite sides of the molecular skeleton (Figure S5). To investigate the aromaticity of compound 4, we performed its NICS analysis. ${ }^{54}$ Surprisingly, the hexagons and most of the heptagons are rather nonaromatic, while the pentagons are highly aromatic, especially the one in the aceheptalene subunit ( $-78 \mathrm{ppm})$ (Figure $4 a)$. The value is even lower than that of pristine aceheptalene (Figure S6a). This scenario is further supported by significant diamagnetic and paramagnetic ring currents on the pentagons and the heptalene subunit, respectively, as
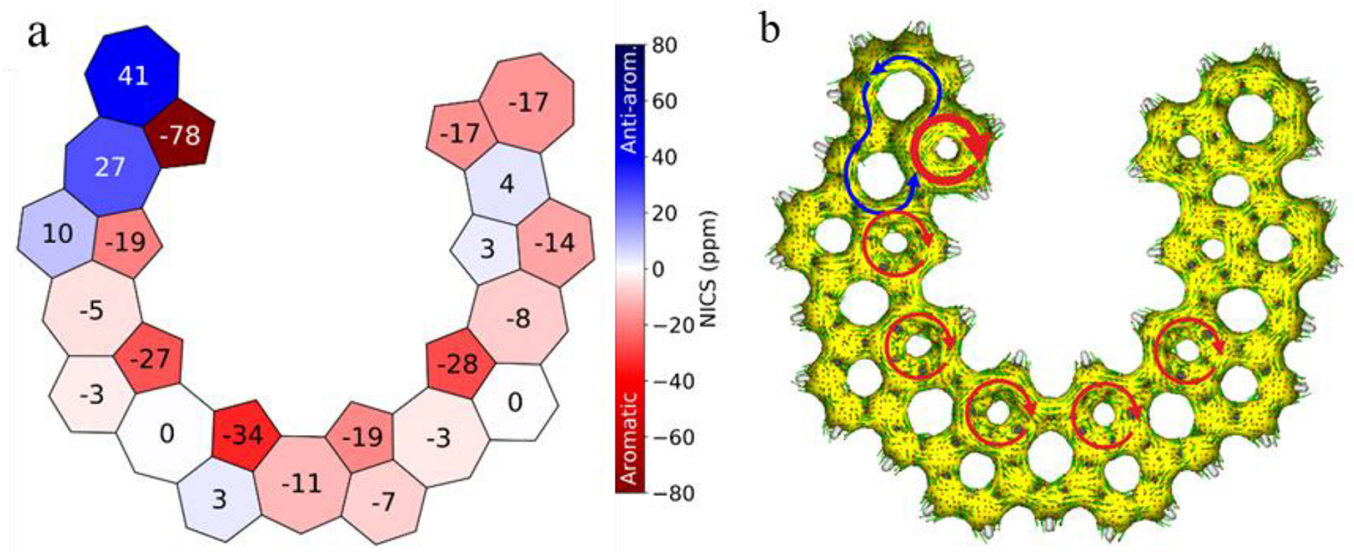

Figure 4. (a) $\operatorname{NICS}_{z z}(1)$ pattern and (b) ACID analysis of 4. NICS is estimated at $1 \AA$ above the center of each ring. The ACID isosurface is shown at an isovalue of 0.05 . Diamagnetic currents and paramagnetic currents are indicated by red and blue arrows, respectively. Calculations were performed at the B3LYP/6-311+G** level of theory. 
suggested by the corresponding ACID analysis ${ }^{55}$ (Figure $4 b$ ). Notably, the $\operatorname{NICS}_{z z}(1)$ values of the pentagons in $\mathbf{4}$ (Figure $4 a)$ are generally lower than those in the reported azuleneembedding PAHs, ${ }^{56-60}$ and close to that of the cyclopentadienide anion ( $-34 \mathrm{ppm}$, Figure S6d), supporting an uneven distribution of electron density in 4.

In conclusion, we developed an on-surface synthesis of polycyclic $\mathrm{sp}^{2}$-carbon nanostructures with multiple regularly fused pentagon-heptagon pairs. Our approach utilized a surface-assisted reaction of a 1,1'-biazulenyl-3,3'-diyl-gold organometallic polymer involving a rearrangement of the azulene skeleton. NICS and ACID analysis suggested a polar electronic structure for these unprecedented $\mathrm{sp}^{2}$-carbon nanostructures. Our results can pave the way for new $\mathrm{sp}^{2}$ carbon frameworks with nonhexagons. Investigation of their potentially intriguing properties, such as an open-shell electronic structure, will be promising for spintronic applications. $^{21,34,61}$ Furthermore, the precise construction of multiple fused pentagon-heptagon pairs can also facilitate the understanding of topologically nontrivial defects in graphene. ${ }^{6}$

\section{ASSOCIATED CONTENT}

\section{SI Supporting Information}

The Supporting Information is available free of charge at https://pubs.acs.org/doi/10.1021/jacs.0c03635.

Experimental methods; characterization data for new compounds; complementary STM, nc-AFM, and calculation figures; proposed reaction mechanism scheme (PDF)

\section{AUTHOR INFORMATION}

\section{Corresponding Authors}

Akimitsu Narita - Max Planck Institute for Polymer Research, 55128 Mainz, Germany; Organic and Carbon Nanomaterials Unit, Okinawa Institute of Science and Technology Graduate University, Kunigami, Okinawa 904-0495, Japan; ๑1 orcid.org/0000-0002-3625-522X; Email: narita@mpipmainz.mpg.de

Roman Fasel - Empa, Swiss Federal Laboratories for Materials Science and Technology, 8600 Dübendorf, Switzerland; Department of Chemistry and Biochemistry, University of Bern, 3012 Bern, Switzerland; 이이이.org/0000-0002-1553-6487; Email: roman.fasel@empa.ch

Klaus Müllen - Max Planck Institute for Polymer Research, 55128 Mainz, Germany; Department Chemie, Johannes Gutenberg-University Mainz, 55128 Mainz, Germany; Email: muellen@mpip-mainz.mpg.de

\section{Authors}

Ian Cheng-Yi Hou - Max Planck Institute for Polymer Research, 55128 Mainz, Germany; Department Chemie, Johannes Gutenberg-University Mainz, 55128 Mainz, Germany; 이이.org/0000-0002-1949-1124

Qiang Sun - Empa, Swiss Federal Laboratories for Materials Science and Technology, 8600 Dübendorf, Switzerland; (D) orcid.org/0000-0003-4903-4570

Kristjan Eimre - Empa, Swiss Federal Laboratories for Materials Science and Technology, 8600 Dübendorf, Switzerland; 이이.org/0000-0002-3444-3286

Marco Di Giovannantonio - Empa, Swiss Federal Laboratories for Materials Science and Technology, 8600 Dübendorf, Switzerland
José I. Urgel - Empa, Swiss Federal Laboratories for Materials Science and Technology, 8600 Dübendorf, Switzerland; (1) orcid.org/0000-0001-7608-2979

Pascal Ruffieux - Empa, Swiss Federal Laboratories for Materials Science and Technology, 8600 Dübendorf, Switzerland; 이이.org/0000-0001-5729-5354

Complete contact information is available at: https://pubs.acs.org/10.1021/jacs.0c03635

\section{Author Contributions}

${ }^{\#}$ I.C.-Y.H. and Q.S. contributed equally.

\section{Notes}

The authors declare no competing financial interest.

\section{ACKNOWLEDGMENTS}

We are thankful for the financial support from the Max Planck Society, the EC through the Marie Sklodowska-Curie ITN project iSwitch (GA-642196), the Swiss National Science Foundation under Grant No. 200020 182015 and No. 200021_172527, and the NCCR MARVEL funded by the Swiss National Science Foundation (51NF40-182892). Computational support from the ETH Zurich HPC cluster Euler is gratefully acknowledged.

\section{REFERENCES}

(1) Cockayne, E.; Rutter, G. M.; Guisinger, N. P.; Crain, J. N.; First, P. N.; Stroscio, J. A. Grain Boundary Loops in Graphene. Phys. Rev. B: Condens. Matter Mater. Phys. 2011, 83 (19), 195425.

(2) Huang, P. Y.; Ruiz-Vargas, C. S.; Van Der Zande, A. M.; Whitney, W. S.; Levendorf, M. P.; Kevek, J. W.; Garg, S.; Alden, J. S.; Hustedt, C. J.; Zhu, Y.; Park, J.; McEuen, P. L.; Muller, D. A. Grains and Grain Boundaries in Single-Layer Graphene Atomic Patchwork Quilts. Nature 2011, 469 (7330), 389-392.

(3) Rasool, H. I.; Ophus, C.; Klug, W. S.; Zettl, A.; Gimzewski, J. K. Measurement of the Intrinsic Strength of Crystalline and Polycrystalline Graphene. Nat. Commun. 2013, 4 (1), 2811.

(4) Yazyev, O. V.; Chen, Y. P. Polycrystalline Graphene and Other Two-Dimensional Materials. Nat. Nanotechnol. 2014, 9 (10), 755767.

(5) Balasubramanian, K.; Biswas, T.; Ghosh, P.; Suran, S.; Mishra, A.; Mishra, R.; Sachan, R.; Jain, M.; Varma, M.; Pratap, R.; Raghavan, $\mathrm{S}$. Reversible Defect Engineering in Graphene Grain Boundaries. Nat. Commun. 2019, 10 (1), 1090.

(6) Banhart, F.; Kotakoski, J.; Krasheninnikov, A. V. Structural Defects in Graphene. ACS Nano 2011, 5 (1), 26-41.

(7) Tsen, A. W.; Brown, L.; Levendorf, M. P.; Ghahari, F.; Huang, P. Y.; Havener, R. W.; Ruiz-Vargas, C. S.; Muller, D. A.; Kim, P.; Park, J. Tailoring Electrical Transport Across Grain Boundaries in Polycrystalline Graphene. Science 2012, 336 (6085), 1143-1146.

(8) Ma, T.; Liu, Z.; Wen, J.; Gao, Y.; Ren, X.; Chen, H.; Jin, C.; Ma, X.-L.; Xu, N.; Cheng, H.-M.; Ren, W. Tailoring the Thermal and Electrical Transport Properties of Graphene Films by Grain Size Engineering. Nat. Commun. 2017, 8 (1), 14486.

(9) Chen, J.-H.; Autès, G.; Alem, N.; Gargiulo, F.; Gautam, A.; Linck, M.; Kisielowski, C.; Yazyev, O. V.; Louie, S. G.; Zettl, A. Controlled Growth of a Line Defect in Graphene and Implications for Gate-Tunable Valley Filtering. Phys. Rev. B: Condens. Matter Mater. Phys. 2014, 89 (12), 121407.

(10) Wei, Y.; Wu, J.; Yin, H.; Shi, X.; Yang, R.; Dresselhaus, M. The Nature of Strength Enhancement and Weakening by PentagongHeptagon Defects in Graphene. Nat. Mater. 2012, 11 (9), 759-763.

(11) Serov, A. Y.; Ong, Z.-Y.; Pop, E. Effect of Grain Boundaries on Thermal Transport in Graphene. Appl. Phys. Lett. 2013, 102 (3), 033104 . 
(12) Zhang, H.; Lee, G.; Gong, C.; Colombo, L.; Cho, K. Grain Boundary Effect on Electrical Transport Properties of Graphene. J. Phys. Chem. C 2014, 118 (5), 2338-2343.

(13) Zhang, Z.; Yang, Y.; Xu, F.; Wang, L.; Yakobson, B. I. Unraveling the Sinuous Grain Boundaries in Graphene. Adv. Funct. Mater. 2015, 25 (3), 367-373.

(14) Yazyev, O. V.; Louie, S. G. Electronic Transport in Polycrystalline Graphene. Nat. Mater. 2010, 9 (10), 806-809.

(15) Gunlycke, D.; White, C. T. Graphene Valley Filter Using a Line Defect. Phys. Rev. Lett. 2011, 106 (13), 136806.

(16) Steer, R. P. Photophysics of Molecules Containing Multiples of the Azulene Carbon Framework. J. Photochem. Photobiol., C 2019, 40, 68-80.

(17) Yoshida, Z.; Shibata, M.; Ogino, E.; Sugimoto, T. A New CataCondensed Nonalternant Tetracyclic Hydrocarbon Azuleno[2,1e]Azulene. Tetrahedron Lett. 1984, 25 (31), 3343-3346.

(18) Takayasu, T.; Nitta, M. On the Reaction of Azulen-2ylmethylene(triphenyl)phosphorane. Convenient Preparation of Azuleno[1,2-f]- and Azuleno[1,2-a]-azulenes and Their Properties. J. Chem. Soc., Perkin Trans. 1 1997, 23 (23), 3537-3542.

(19) Anderson, A. G.; Montana, A. F.; MacDonald, A.; Masada, G. M. Synthesis of Dicyclopenta[ef,kl]heptalene (Azupyrene). II. Routes from 1,6,7,8,9,9a-Hexahydro-2H-Benzo[c,d] azulen-6-one and 5-Phenylpentanoic Acid. J. Org. Chem. 1973, 38 (8), 1445-1450.

(20) Hafner, K.; Diehl, H.; Richarz, W. Cycloadditions of Aceheptylene-A Facile Synthesis of Dicyclopenta[ef,kl]heptalenes. Angew. Chem., Int. Ed. Engl. 1976, 15 (2), 108-109.

(21) Konishi, A.; Horii, K.; Shiomi, D.; Sato, K.; Takui, T.; Yasuda, M. Open-Shell and Antiaromatic Character Induced by the Highly Symmetric Geometry of the Planar Heptalene Structure: Synthesis and Characterization of a Nonalternant Isomer of Bisanthene. J. Am. Chem. Soc. 2019, 141 (26), 10165-10170.

(22) Reel, H.; Vogel, E. Dicyclohepta $[c d, g h]$ Pentalene-A New Pyrene Isomer. Angew. Chem., Int. Ed. Engl. 1972, 11 (11), 10131014.

(23) Vogel, E.; Wieland, H.; Schmalstieg, L.; Lex, J. Novel Synthesis and Molecular Structure of the Pyrene Isomer Dicyclohepta $[c d, g h]$ pentalene (Azuleno[2,1,8-ija]azulene). Angew. Chem., Int. Ed. Engl. 1984, 23 (9), 717-719.

(24) Han, Y.; Xue, Z.; Li, G.; Gu, Y.; Ni, Y.; Dong, S.; Chi, C. Formation of Azulene-Embedded Nanographene: Naphthalene to Azulene Rearrangement During the Scholl Reaction. Angew. Chem., Int. Ed. 2020, Accepted Author Manuscript, DOI: 10.1002/ anie.201915327.

(25) Sun, Q.; Zhang, R.; Qiu, J.; Liu, R.; Xu, W. On-Surface Synthesis of Carbon Nanostructures. Adv. Mater. 2018, 30 (17), 1705630.

(26) Gröning, O.; Wang, S.; Yao, X.; Pignedoli, C. A.; Borin Barin, G.; Daniels, C.; Cupo, A.; Meunier, V.; Feng, X.; Narita, A.; Müllen, $\mathrm{K}$. Engineering of Robust Topological Quantum Phases in Graphene Nanoribbons. Nature 2018, 560 (7717), 209-213.

(27) Rizzo, D. J.; Veber, G.; Cao, T.; Bronner, C.; Chen, T.; Zhao, F.; Rodriguez, H.; Louie, S. G.; Crommie, M. F.; Fischer, F. R. Topological Band Engineering of Graphene Nanoribbons. Nature 2018, 560 (7717), 204-208.

(28) Di Giovannantonio, M.; Urgel, J. I.; Beser, U.; Yakutovich, A. V.; Wilhelm, J.; Pignedoli, C. A.; Ruffieux, P.; Narita, A.; Müllen, K.; Fasel, R. On-Surface Synthesis of Indenofluorene Polymers by Oxidative Five-Membered Ring Formation. J. Am. Chem. Soc. 2018, 140 (10), 3532-3536.

(29) Mishra, S.; Beyer, D.; Eimre, K.; Kezilebieke, S.; Berger, R.; Gröning, O.; Pignedoli, C. A.; Müllen, K.; Liljeroth, P.; Ruffieux, P.; Feng, X.; Fasel, R. Topological Frustration Induces Unconventional Magnetism in a Nanographene. Nat. Nanotechnol. 2020, 15 (1), 2228.

(30) Ruffieux, P.; Wang, S.; Yang, B.; Sánchez-Sánchez, C.; Liu, J.; Dienel, T.; Talirz, L.; Shinde, P.; Pignedoli, C. A.; Passerone, D.; Dumslaff, T.; Feng, X.; Müllen, K.; Fasel, R. On-Surface Synthesis of
Graphene Nanoribbons with Zigzag Edge Topology. Nature 2016, 531 (7595), 489-492.

(31) Clair, S.; De Oteyza, D. G. Controlling a Chemical Coupling Reaction on a Surface: Tools and Strategies for On-Surface Synthesis. Chem. Rev. 2019, 119 (7), 4717-4776.

(32) Grill, L.; Hecht, S. Covalent On-Surface Polymerization. Nat. Chem. 2020, 12 (2), 115-130.

(33) Mishra, S.; Lohr, T. G.; Pignedoli, C. A.; Liu, J.; Berger, R.; Urgel, J. I.; Müllen, K.; Feng, X.; Ruffieux, P.; Fasel, R. Tailoring Bond Topologies in Open-Shell Graphene Nanostructures. ACS Nano 2018, 12 (12), 11917-11927.

(34) Liu, J.; Mishra, S.; Pignedoli, C. A.; Passerone, D.; Urgel, J. I.; Fabrizio, A.; Lohr, T. G.; Ma, J.; Komber, H.; Baumgarten, M.; Corminboeuf, C.; Berger, R.; Ruffieux, P.; Müllen, K.; Fasel, R.; Feng, X. Open-Shell Nonbenzenoid Nanographenes Containing Two Pairs of Pentagonal and Heptagonal Rings. J. Am. Chem. Soc. 2019, 141 (30), 12011-12020.

(35) Hieulle, J.; Carbonell-Sanromà, E.; Vilas-Varela, M.; GarciaLekue, A.; Guitián, E.; Peña, D.; Pascual, J. I. On-Surface Route for Producing Planar Nanographenes with Azulene Moieties. Nano Lett. 2018, 18 (1), 418-423.

(36) Krug, C. K.; Nieckarz, D.; Fan, Q.; Szabelski, P.; Gottfried, M. The Macrocycle Versus Chain Competition in On-Surface Polymerization: Insights from Reactions of 1,3-Dibromoazulene on $\mathrm{Cu}(111)$. Chem. - Eur. J. 2020, Accepted Author Manuscript, DOI: 10.1002/ chem.202000486.

(37) Krug, C. K.; Fan, Q.; Fillsack, F.; Glowatzki, J.; Trebel, N.; Heuplick, L. J.; Koehler, T.; Gottfried, J. M. Organometallic Ring vs. Chain Formation beyond Kinetic Control: Steering Their Equilibrium in Two-Dimensional Confinement. Chem. Commun. 2018, 54 (70), 9741-9744.

(38) Fan, Q.; Martin-Jimenez, D.; Ebeling, D.; Krug, C. K.; Brechmann, L.; Kohlmeyer, C.; Hilt, G.; Hieringer, W.; Schirmeisen, A.; Gottfried, J. M. Nanoribbons with Nonalternant Topology from Fusion of Polyazulene: Carbon Allotropes beyond Graphene. J. Am. Chem. Soc. 2019, 141 (44), 17713-17720.

(39) Sun, Q.; Hou, I. C.-Y.; Eimre, K.; Pignedoli, C. A.; Ruffieux, P.; Narita, A.; Fasel, R. On-Surface Synthesis of Polyazulene with 2,6Connectivity. Chem. Commun. 2019, 55 (89), 13466-13469.

(40) Shiotari, A.; Nakae, T.; Iwata, K.; Mori, S.; Okujima, T.; Uno, H.; Sakaguchi, H.; Sugimoto, Y. Strain-Induced Skeletal Rearrangement of a Polycyclic Aromatic Hydrocarbon on a Copper Surface. Nat. Commun. 2017, 8 (1), 16089.

(41) Wang, F.; Lai, Y. H.; Kocherginsky, N. M.; Kosteski, Y. Y. The First Fully Characterized 1,3-Polyazulene: High Electrical Conductivity Resulting from Cation Radicals and Polycations Generated upon Protonation. Org. Lett. 2003, 5 (7), 995-998.

(42) Dubovik, J.; Bredihhin, A. A Convenient Synthesis of Functionalized Azulenes via Negishi Cross-Coupling. Synthesis 2015, 47 (04), 538-548.

(43) Shang, J.; Wang, Y.; Chen, M.; Dai, J.; Zhou, X.; Kuttner, J.; Hilt, G.; Shao, X.; Gottfried, J. M.; Wu, K. Assembling Molecular Sierpiński Triangle Fractals. Nat. Chem. 2015, 7 (5), 389-393.

(44) This refers to a $1,1^{\prime}$-biazulenyl moiety with substitutions on its $3,3^{\prime}$-positions.

(45) Urgel, J. I.; Hayashi, H.; Di Giovannantonio, M.; Pignedoli, C. A.; Mishra, S.; Deniz, O.; Yamashita, M.; Dienel, T.; Ruffieux, P.; Yamada, H.; Fasel, R. On-Surface Synthesis of Heptacene Organometallic Complexes. J. Am. Chem. Soc. 2017, 139 (34), 11658-11661.

(46) Heilbronner, E.; Plattner, P. A.; Wieland, K. Umlagerung von Azulen in Naphthalin. Experientia 1947, 3 (2), 70.

(47) Scott, L. T.; Agopian, G. K. Automerization of Naphthalene. J. Am. Chem. Soc. 1977, 99 (13), 4506-4507.

(48) Parent, J. F.; Deslongchamps, P. High-Temperature Isomerization of Benzenoid Polycyclic Aromatic Hydrocarbons. Analysis through the Bent Bond and Antiperiplanar Hypothesis Orbital Model. J. Org. Chem. 2018, 83 (6), 3299-3304. 
(49) Wentrup, C.; Becker, J. Azulene-Naphthalene-Type Rearrangements in Benz $[a]$ azulene and Cyclohepta $[b]$ indole. Chem. - Eur. J. 2016, 22 (39), 13835-13839.

(50) Stirling, A.; Iannuzzi, M.; Laio, A.; Parrinello, M. Azulene-toNaphthalene Rearrangement: The Car-Parrinello Metadynamics Method Explores Various Reaction Mechanisms. ChemPhysChem 2004, 5 (10), 1558-1568.

(51) Klein, B. P.; Harman, S. E.; Ruppenthal, L.; Ruehl, G. M.; Hall, S. J.; Carey, S. J.; Herritsch, J.; Schmid, M.; Maurer, R. J.; Tonner, R.; Campbell, C. T.; Gottfried, J. M. Enhanced Bonding of PentagonHeptagon Defects in Graphene to Metal Surfaces: Insights from the Adsorption of Azulene and Naphthalene to $\mathrm{Pt}(111)$. Chem. Mater. 2020, 32 (3), 1041-1053.

(52) Klein, B. P.; Van Der Heijden, N. J.; Kachel, S. R.; Franke, M.; Krug, C. K.; Greulich, K. K.; Ruppenthal, L.; Müller, P.; Rosenow, P.; Parhizkar, S.; Bocquet, F. C.; Schmid, M.; Hieringer, W.; Maurer, R. J.; Tonner, R.; Kumpf, C.; Swart, I.; Gottfried, J. M. Molecular Topology and the Surface Chemical Bond: Alternant Versus Nonalternant Aromatic Systems as Functional Structural Elements. Phys. Rev. X 2019, 9 (1), 011030.

(53) Alder, R. W.; East, S. P.; Harvey, J. N.; Oakley, M. T. The Azulene-to-Naphthalene Rearrangement Revisited: A DFT Study of Intramolecular and Radical-Promoted Mechanisms. J. Am. Chem. Soc. 2003, 125 (18), 5375-5387.

(54) Chen, Z.; Wannere, C. S.; Corminboeuf, C.; Puchta, R.; v. R. Schleyer, P. NICS as an Aromaticity Criterion. Chem. Rev. 2005, 105 (10), 3842 .

(55) Geuenich, D.; Hess, K.; Köhler, F.; Herges, R. Anisotropy of the Induced Current Density (ACID), a General Method to Quantify and Visualize Electronic Delocalization. Chem. Rev. 2005, 105 (10), 3758-3772.

(56) Ma, J.; Fu, Y.; Dmitrieva, E.; Liu, F.; Komber, H.; Hennersdorf, F.; Popov, A. A.; Weigand, J. J.; Liu, J.; Feng, X. Helical Nanographenes Containing an Azulene Unit: Synthesis, Crystal Structures, and Properties. Angew. Chem., Int. Ed. 2020, 59 (14), $5637-5642$.

(57) Yamamoto, K.; Ie, Y.; Tohnai, N.; Kakiuchi, F.; Aso, Y. Antiaromatic Character of Cycloheptatriene-bis-annelated Indenofluorene Framework Mainly Originated from Heptafulvene Segment. Sci. Rep. 2018, 8 (1), 17663.

(58) Konishi, A.; Morinaga, A.; Yasuda, M. Construction of Polycyclic $\pi$-Conjugated Systems Incorporating an Azulene Unit Following the Oxidation of 1,8-Diphenyl-9,10-bis(phenylethynyl)phenanthrene. Chem. - Eur. J. 2018, 24 (34), 8548-8552.

(59) Zhang, X.; Huang, Y.; Zhang, J.; Meng, W.; Peng, Q.; Kong, R.; Xiao, Z.; Liu, J.; Huang, M.; Yi, Y.; Chen, L.; Fan, Q.; Lin, G.; Liu, Z.; Zhang, G.; Jiang, L.; Zhang, D. Dicyclohepta $[i j k l, u v w x]$ rubicene with Two Pentagons and Two Heptagons as a Stable and Planar Nonbenzenoid Nanographene. Angew. Chem., Int. Ed. 2020, 59 (9), 35293533.

(60) Sasaki, Y.; Takase, M.; Okujima, T.; Mori, S.; Uno, H. Synthesis and Redox Properties of Pyrrole- and Azulene-fused Azacoronene. Org. Lett. 2019, 21 (6), 1900-1903.

(61) Jiang, Q.; Tao, T.; Phan, H.; Han, Y.; Gopalakrishna, T. Y.; Herng, T. S.; Li, G.; Yuan, L.; Ding, J.; Chi, C. Diazuleno-s-Indacene Diradicaloids: Syntheses, Properties, and Local (Anti)Aromaticity Shift from Neutral to Dicationic State. Angew. Chem., Int. Ed. 2018, 57 (51), 16737-16741. 